\title{
Identification of novel urinary biomarkers for assessing disease activity and prognosis of rheumatoid arthritis
}

\author{
Yune-Jung Park ${ }^{1,2}$, Seung-Ah Yoo $^{2}$, Daehee Hwang ${ }^{3}$, Chul-Soo Cho ${ }^{2,4}$ and Wan-Uk Kim²,5 \\ To optimize treatment for rheumatoid arthritis (RA), it is ideal to monitor the disease activity on a daily basis because RA activity \\ fluctuates over time. Urine can be collected routinely at home by patients. Recently, we identified four urinary biomarker \\ candidates_-gelsolin (GSN), orosomucoid (ORM)1, ORM2 and soluble CD14 (SCD14)_in RA patients through transcriptomic \\ and proteomic studies. Here, we investigated the clinical significance of the aforementioned urinary biomarker candidates in a \\ prospective manner. For the first time, we found that urinary ORM1, ORM2 and SCD14 levels, but not GSN, were elevated in RA \\ patients and had a positive correlation with the status of the disease activity. In particular, urine tests for ORM 1, ORM 2 and \\ sCD14 efficiently represented the presence of high RA activity without the need for measuring blood markers. In a parallel study, \\ a more rapid radiographic progression over 3 years was observed in patients with higher ORM2 levels. Combined measurements \\ of urinary ORM2 and serum C-reactive protein synergistically increased the predictability of the radiographic progression of RA \\ (odds ratio: 46.5). Collectively, our data provide evidence that blood-free, urinary biomarkers are promising surrogates for \\ assessing disease activity and prognosis of RA. We anticipate that our urinary biomarkers will provide novel candidates for \\ patient-driven measurements of RA activity at home and can shift the paradigm from blood to urine testing in the assessment \\ of RA activity and prognosis in hospitals.
}

Experimental \& Molecular Medicine (2016) 48, e211; doi:10.1038/emm.2015.120; published online 26 February 2016

\section{INTRODUCTION}

Rheumatoid arthritis (RA) is a chronic inflammatory disease that progressively destroys cartilage and bone. The assessment of the status of RA is a central aspect of patient care. The reduction of RA activity is the major target of therapeutic interventions. ${ }^{1}$ Erythrocyte sedimentation rate (ESR) and C-reactive protein (CRP) are the most commonly used blood markers to evaluate disease activity of RA. However, ESR stays elevated for some time after inflammation is reduced by treatments, and it can be influenced by anemia and age.,3 CRP is non-specifically influenced by infection. Importantly, ESR and CRP may remain normal in more than a third of RA patients at presentation irrespective of disease activity. ${ }^{4,5}$ The disease activity score 28 (DAS28), which is based on counts of tender and swollen joints and ESR (or CRP) measurements, has been widely used for RA assessment. However, it requires specific expertise. Anti-cyclic citrullinated peptide antibody (ACPA), a representative diagnostic marker of RA, can help predict radiographic progression. However, ACPA does not usually correlate with disease activity of RA. ${ }^{6}$ In these instances, there remains an unmet need for alternative biomarkers that can complement conventional serum measures to precisely monitor disease activity of RA.

Another important issue in assessing RA activity is that there is no daily-based measure of activity, such as exists for glucose or blood pressure monitoring. It is well known that mean disease activity assessed by ESR and CRP at regular intervals is the major determinant in predicting joint damage and extraarticular complications associated with RA, ${ }^{1}$ including cardiovascular diseases and osteoporosis. In addition, cumulative levels of DAS28 correlate well with RA progression and complications. ${ }^{1}$ Thus, maintaining a minimal residual level of disease activity (DAS28 below 2.6), as suggested by the targetto-treat strategy, is critical for the optimal treatment of RA. ${ }^{1}$ To optimize the treatment for RA, it would be ideal to monitor RA activity on a daily basis, as can be done for blood pressure

\footnotetext{
${ }^{1}$ Division of Rheumatology, Department of Internal Medicine, St Vincent's Hospital, College of Medicine, Catholic University of Korea, Suwon, Korea; ${ }^{2}$ POSTECH-CATHOLIC Biomedical Engineering Institute, Catholic University of Korea, Seoul, Korea; ${ }^{3}$ Department of New Biology and Center for Plant Aging Research, Institute for Basic Science, DGIST, Daegu, Korea; ${ }^{4}$ Division of Rheumatology, Department of Internal Medicine, Yeouido St Mary's Hospital, College of Medicine, Catholic University of Korea, Seoul, Korea and ${ }^{5}$ Division of Rheumatology, Department of Internal Medicine, Seoul St Mary's Hospital, College of Medicine, Catholic University of Korea, Seoul, Korea

Correspondence: Professor W-U Kim, Division of Rheumatology, Department of Internal Medicine, Seoul St Mary's Hospital, College of Medicine, Catholic University of Korea, 222 Banpo-daero, 137-701 Seoul, Republic of Korea.
}

E-mail: wan725@catholic.ac.kr

Received 11 October 2015; revised 13 November 2015; accepted 23 November 2015 
and glucose, because the activity of RA fluctuates over time. We could then titrate anti-rheumatic medications according to the mean disease activity. However, in clinical practice, most rheumatologists cannot consistently monitor RA activity on a daily or weekly basis when patients only visit hospitals every 1-2 months. Another reason for this may be the lack of time during outpatient visits and the lack of simple reliable biomarkers to perform this assessment. If patients could periodically perform self-diagnostic tests for surrogate markers of RA activity at home without visiting the hospital, cumulative or multiple test results may more accurately reflect RA activity than a single set of measurements taken at the hospital. In addition, this would enable doctors to more tightly control RA progression and complications.

Urinary biomarkers for RA may be a way to address this issue. Urine can be collected routinely at home by patients. Urinary biomarkers provide a complementary dimension to serum measures for some diseases that frequently involve the kidney, including diabetes mellitus and systemic lupus erythematosus. ${ }^{7,8}$ Recently, we identified four urinary biomarker candidates-gelsolin (GSN), orosomucoid (ORM)1, ORM2 and soluble CD14 (sCD14) - in RA patients through the integration of differentially expressed proteins with gene expression data from joint tissues and peripheral blood mononuclear cells. ${ }^{9}$ In a subsequent proof-of-concept trial, we also demonstrated that urinary sCD14 levels were predictive of RA activity. ${ }^{9}$ In the present study, we investigated the clinical significance of the four urinary biomarker candidates in a prospective manner, focusing on their role in assessing RA activity and prognosis.

\section{MATERIALS AND METHODS}

\section{Patients and controls}

Two hundred sixty-four patients with RA and one hundred eightseven control subjects were consecutively recruited from outpatients at our hospital. All RA patients fulfilled the 1987 American College of Rheumatology criteria for RA. ${ }^{10}$ Urine samples were obtained from patients with RA and non-RA controls. Non-RA controls were recruited from healthy subjects without inflammatory arthritis. The following subjects were excluded: those with severe cardiac diseases, renal diseases that could affect glomerular filtration rate, current or chronic infections, pregnancy or a history of malignancy. The study protocol was approved by the Institutional Review Board of the Catholic Medical Center (VC12RISI0191). All patients gave written informed consent to the study protocol.

\section{Determination of RA activity and severity}

Disease activity of RA was evaluated with the DAS2 $8_{\mathrm{ESR}} \cdot{ }^{11}$ The severity of RA was assessed by evaluating radiographic damage on X-rays of the hands and feet of subjects. Radiographs of the hands and feet were taken at baseline and annually thereafter. Radiographic severity was scored in chronologic order for erosions and joint space narrowing according to the Sharp/van der Heijde method. ${ }^{12}$ Severity scores were determined by two board-certified physicians who were blinded to each patient's identity or clinical status. The inter-observer variability described by the interclass correlation coefficient was 0.91 . Joint space narrowing and erosion scores were summed to give a total radiographic progression score. Erosion and narrowing progression scores were calculated by subtracting the initial score from the score after a 3 -year follow-up. Radiographic progression was defined as a progression score $\geqslant 4 .{ }^{13}$

\section{ELISA}

Urine samples were collected from the first or second urination of the day into sterile $500 \mathrm{ml}$ plastic tubes containing $0.05 \%$ sodium azide. Urine samples were centrifuged at $14000 \mathrm{~g}$ for $10 \mathrm{~min}$ at $4{ }^{\circ} \mathrm{C}$ immediately after collection. The clarified supernatants were stored at $-70{ }^{\circ} \mathrm{C}$ until used for analysis. Urinary concentrations of sCD14, GSN, ORM1 and ORM2 were also determined by ELISA, as described previously. ${ }^{9}$

\section{Statistical analysis}

Comparisons between the two groups were performed by a Mann-Whitney $U$-test. A $\chi^{2}$ test was used to compare proportions. Spearman correlation coefficients were used to describe the association between two variables. Radiographic progression events (any increase in an Sharp/van der Heijde score $\geqslant 4$ ) were investigated to determine the presence of radiographic progression after 3 years. To evaluate the ability of urinary proteins to predict high disease activity $(\mathrm{DAS} 28>5.1)$ and radiographic progression, receiver operating characteristic (ROC) curves were plotted. We also tested whether combined urinary measurements, such as ORM1+ORM2, ORM1 $+\mathrm{sCD} 14$ and ORM2+sCD14, had different predictive powers for disease activity. For this, positive values for ORM1, ORM2 and sCD14 were defined as those above the cutoff levels of $0.34,0.15$ and $22.4 \mathrm{ng} \mathrm{ml}^{-1}$, respectively. Optimal cutoff levels for urinary proteins were determined by ROC curves. The number of patients with both positive marker values and high disease activity scores (DAS28 > 5.1) was 54, 61 and 63 for ORM1, ORM2 and sCD14, respectively. The number of patients who were double-positive for two urinary proteins was 46, 49 and 51 for ORM1 and ORM2, ORM1 and sCD14, and ORM2 and sCD14, respectively. The number of patients who were double-negative for two urinary proteins was 39, 57 and 50 for ORM1 and ORM2, ORM1 and sCD14, and ORM2 and sCD14, respectively. Probability plots, which were based on serum CRP levels in patients who had higher urinary ORM2 levels $\left(\geqslant 2.21 \mathrm{ng} \mathrm{ml}^{-1}\right.$, the highest tertile) versus lower urinary ORM2 levels $\left(<2.21 \mathrm{ng} \mathrm{ml}^{-1}\right.$ ), were used to display radiographic progression. A multivariate logistic regression analysis was performed to determine significant and independent contributions of specific variables to the radiographic progression. Multivariate models included all covariates with associations from the univariate models with a $P$ value $\leqslant 0.20$. All reported $P$ values were two-tailed, with a $P$ value $<0.05$ indicating statistical significance.

\section{RESULTS}

\section{Characteristics of study population}

Baseline characteristics of the 264 RA patients and 187 non-RA controls are shown in Supplementary Table 1. The mean ages of RA patients and non-RA controls were 53.2 and 52.9 years, respectively. The median glomerular filtration rate in RA patients was higher than that in control subjects. No significant difference was found in sex, serum creatinine, percentage of patients with hypertension or percentage with diabetes mellitus between the two groups. A total of 173 (65.5\%) patients received methotrexate, $194(73.4 \%)$ received hydroxychloroquine, $26(9.8 \%)$ received anti-tumor necrosis factor- $\alpha$ 

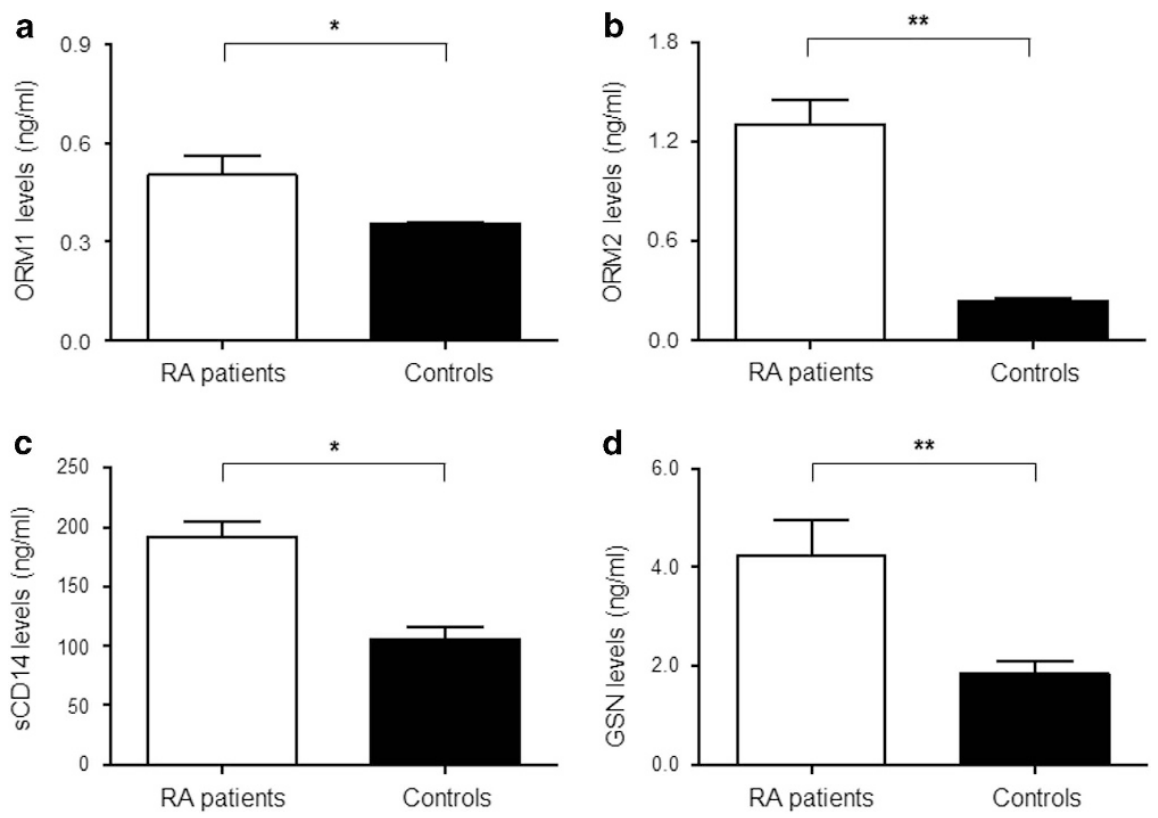

Figure 1 Comparison of urinary levels of biomarker candidates between patients with rheumatoid arthritis (RA) and non-RA controls. Levels of four urine proteins, including (a) orosomucoid (ORM) 1, (b) ORM2, (c) soluble CD14 (SCD14) and (d) gelsolin (GSN) were determined in the urine of RA patients $(n=264)$ and non-RA controls $(n=187)$ by ELISA. The data are presented as the mean \pm standard error of mean (s.e.m.). ${ }^{*} P<0.05$ and ${ }^{* *} P<0.01$.

(anti-TNF- $\alpha$ ) therapy and $198(75.0 \%)$ were treated with low-dose prednisolone ( $\leqslant 10 \mathrm{mg}$ per day).

\section{Correlation of urinary protein levels with RA activity} Urinary levels of four proteins (GSN, ORM1, ORM2 and sCD14), after adjustment for urine creatinine, in RA patients $(n=264)$ were significantly higher than those in control subjects $(n=187$; Figure 1a-d). There was no difference in the four urinary protein levels in relation to the use of medications, including prednisolone, methotrexate, hydroxychloroquine and anti-TNF- $\alpha$ therapy (data not shown). To assess the clinical significance of urinary proteins, we examined the association of the four proteins with several parameters representing disease activity of RA. When we divided RA patients into three groups based on DAS28 results (low: DAS $28 \leqslant 3.2(n=76)$, moderate: $3.2<$ DAS $28 \leqslant 5.1 \quad(n=114)$ and high: DAS28 $>5.1(n=74))$, urinary ORM1, ORM2 and sCD14 levels increased in proportion to DAS28 (Figure 2a) values. In univariate analyses, ORM1, ORM2 and sCD14 levels were positively correlated with blood inflammatory marker levels, such as ESR and CRP (Figure 2b). Particularly, ORM2 showed the highest correlation co-efficiency with CRP $(r=0.55)$, which was similar to the correlation between ESR and CRP $(r=0.54)$. Moreover, urinary ORM2 was positivity correlated with urinary sCD14 $(r=0.37)$, a well-known serum biomarker of RA activity. ${ }^{14,15}$ However, ORM1 showed a weak correlation with ESR and CRP (Figure 2b). Interestingly, an analysis of clustering correlations showed different patterns between RA and non-RA controls, particularly in the correlations of ORM1 with ORM2 and sCD14 (Supplementary Figure 1).
Next, we compared the diagnostic power of four urinary proteins for predicting high disease activity (DAS28 $>5.1$ ) with the diagnostic power of conventional markers for chronic inflammation, including CRP, hemoglobin and albumin. When we analyzed ROC curves of models, the area under the curve (AUC) of sCD14 was 0.59 (95\% confidence interval (CI): $0.47-0.65)$ and that of ORM1 was $0.61(0.47-0.63)$. The AUC of ORM2 was $0.66(0.56-0.74)$, which was comparable to that of CRP (0.66 (0.57-0.73); Figure 2c). However, GSN had no significant association with high disease activity (Figure 2c). No significant difference in AUC was found between OMR2 and CRP levels, suggesting that urine ORM2 could replace serum CRP in predicting high RA activity. Moreover, when two urinary biomarkers were simultaneously analyzed as a combined measure of activity, their sensitivity and specificity for predicting high disease activity were better than those when using a single urinary marker (Figure 2d). Particularly, for RA patients with elevated ORM1 and ORM2 in their urine, sensitivity and specificity for predicting high activity were $98 \%$ and $76 \%$, respectively. These results suggest that urinary tests using one or two biomarkers out of ORM1, ORM2 and sCD14 could help clinicians monitor disease activity as efficiently as serum CRP, a conventional inflammatory marker of RA.

\section{ORM2 as a urinary biomarker for RA progression}

It is well known that accumulated disease activity can determine the severity and prognosis of RA. ${ }^{1,16,17}$ Based on our finding that urinary protein levels could reflect disease activity, we further investigated whether these urinary proteins could serve as a predictor of radiographic progression. 
a
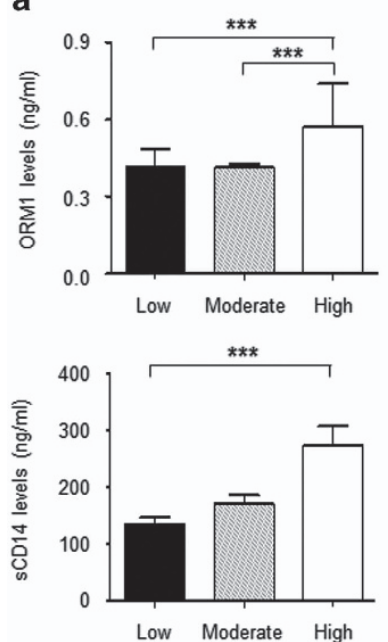

C

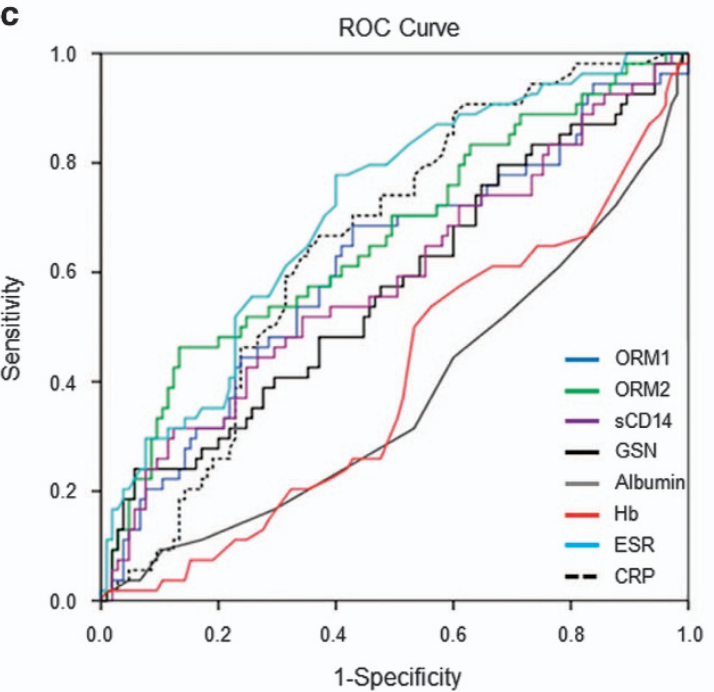

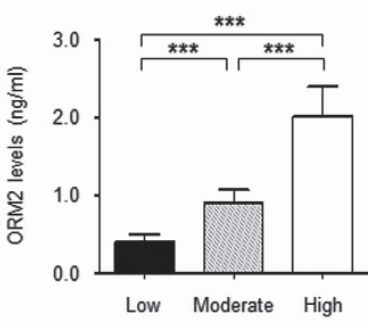

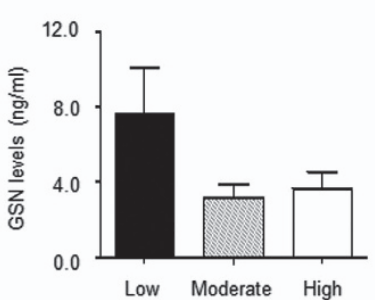

d

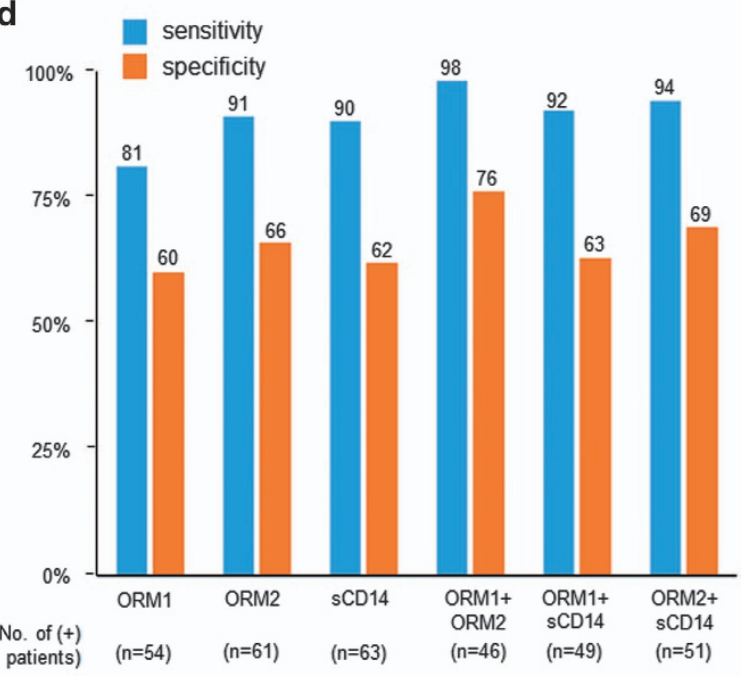

Figure 2 Relationship of urinary ORM1, ORM2 and SCD14 levels with RA activity. (a) Urinary protein (ORM1, ORM2 and sCD14) levels in relation to disease activity of RA. RA activity was assessed with the disease activity score $28_{\text {ESR }}$ (DAS28 $8_{\text {ESR }}$ ) as low activity at DAS28 $\leqslant 3.2(n=76)$, moderate activity at $3.2<$ DAS28 $\leqslant 5.1 \quad(n=114)$, and high activity at DAS28 $>5.1(n=74)$. The data are presented as the mean \pm s.e.m. ${ }^{* *} P<0.001$. (b) Clustering correlations of urinary protein levels with the blood inflammatory markers of RA. ${ }^{*} P<0.05,{ }^{* *} P<0.01$ and ${ }^{* * *} P<0.001$. The power of the correlation is represented by the color and size of the circles. (c) Receiver operating characteristic (ROC) curves analyses to assess the association of high RA activity with urinary biomarker proteins, including ORM1, ORM2 and SCD14. High activity was defined as DAS28 $>5.1$. The area under the curve was 0.61 for ORM1 $(P=0.020), 0.66$ for ORM2 $(P=0.001), 0.57$ for gelsolin (GSN, $P=0.12), 0.59$ for $(P=0.071), 0.37$ for albumin $(P=0.009), 0.40$ for hemoglobin $(\mathrm{Hb}, P=0.040), 0.71$ for ESR $(P<0.001)$ and 0.66 for CRP $(P<0.001)$. (d) Sensitivity and specificity of urinary protein markers for assessing high RA activity (DAS28 $>5.1$ ) when their optimal cutoff values are introduced. The cutoff values were $0.34 \mathrm{ng} \mathrm{ml}^{-1}$ for $\mathrm{ORM} 1$, $0.15 \mathrm{ng} \mathrm{ml}^{-1}$ for ORM2 and $22.4 \mathrm{ng} \mathrm{ml}^{-1}$ for SCD14.

The median value of baseline radiographic scores was 40.9 (interquartile range: 15.7-64.3) and the mean value at a 3-year follow-up was 44.6 (interquartile range: 13.6-69.6). When baseline clinical parameters and urinary protein levels in RA patients with $(n=24)$ or without radiographic progression $(n=240)$ were compared, progressive RA patients had longer disease durations, higher glomerular filtration rate, elevated ESR and CRP levels and more ACPA (Supplementary Table 2), which is in agreement with previous reports. ${ }^{18}$ The group with radiographic progression had higher ORM2 levels than the non-progression group $\left(3.22 \mathrm{ng} \mathrm{ml}^{-1}(0.36-5.42)\right.$ and
$0.26 \mathrm{ng} \mathrm{ml}^{-1}$ (0.17-0.60), respectively, $P<0.001$; Figure $\left.3 \mathrm{a}\right)$. Interestingly, patients with higher urinary ORM2 levels $\left(\geqslant 2.21 \mathrm{ng} \mathrm{ml}^{-1}\right)$ at baseline had more severe radiographic progression after 3 years than those with lower urinary ORM2 levels (<2.21 ng ml${ }^{-1} ; 44.6$ (18.7-62.1) and 38.8 (13.5-69.4), respectively, $P=0.005)$. These data suggest that urinary ORM2 can serve as a surrogate biomarker to predict RA progression.

Multivariate logistic regression analysis was performed to assess whether urinary protein levels could independently predict radiographic progression. Our results showed that urinary ORM2 levels significantly increased the risk of the 
a
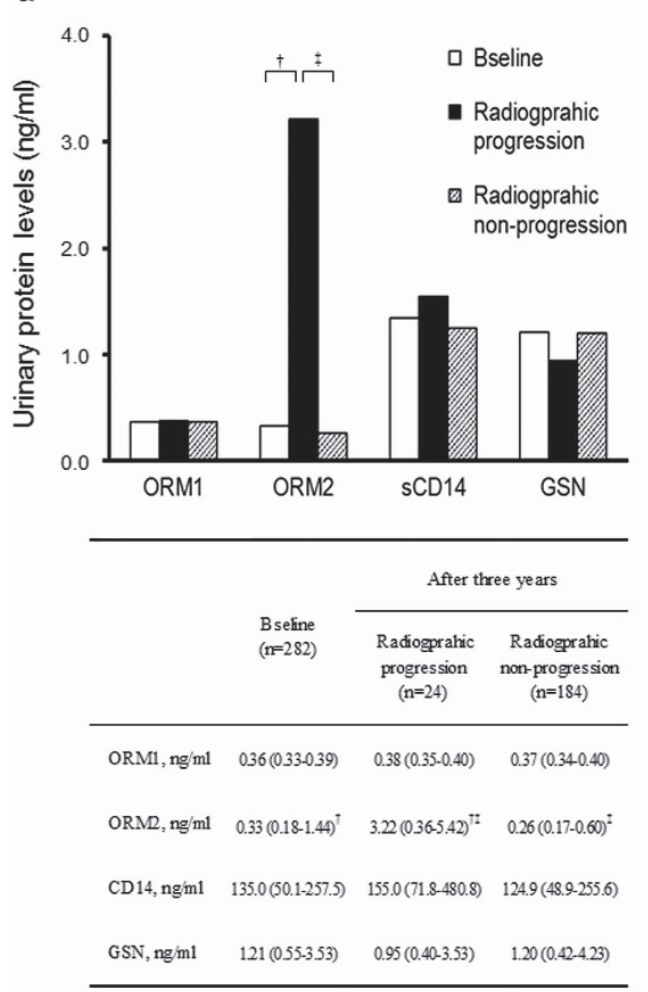

*Data are presented as medain and (interquartile range). ORM denotes alpha-1-acid glycoprotein; GSN is gelsolin TANCOVA was used; $P$ value $<0.001$

tMann-Whitney $U$ test was use $\mathrm{d}$ : $\mathrm{P}$ value $<0.00$ b
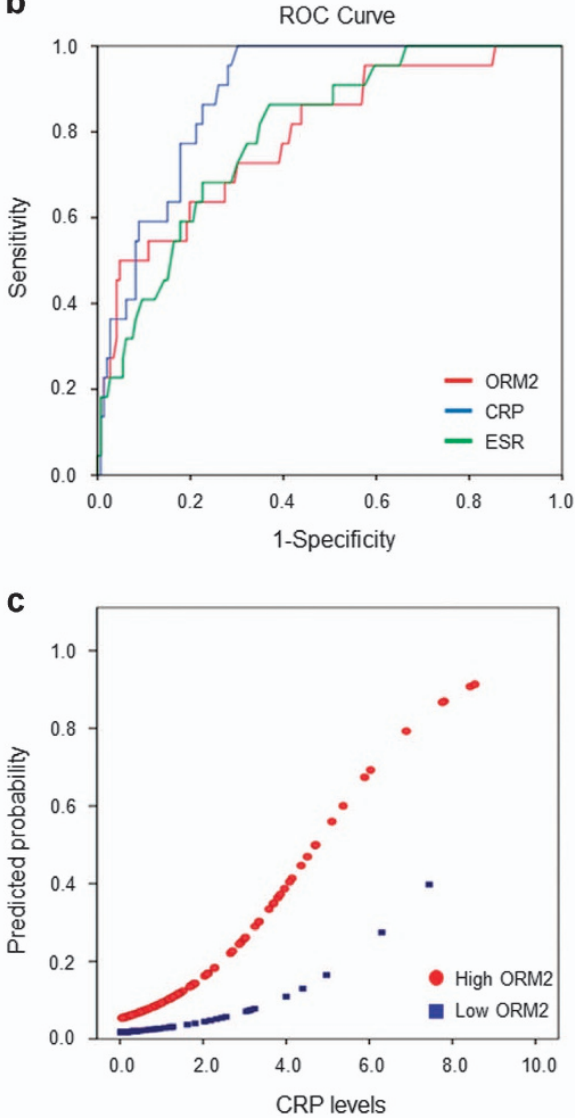

Figure 3 Correlation of urinary ORM2 levels with radiographic progression of RA. (a) Urinary levels of ORM1, ORM2 and sCD14 in relation to the radiographic severity of RA. Radiographic severity was assessed by evaluating radiographic damage on X-rays of the hands and feet, which were taken at baseline and annually thereafter. Radiographic progression was defined as a progression score $\geqslant 4$. (b) ROC curve analysis for radiographic progression revealed that ORM2 had an area under the curve (AUC) comparable to those of ESR and CRP. The AUC was 0.79 for ORM2 $(P<0.001), 0.81$ for ESR $(P<0.001)$ and 0.89 for CRP $(P<0.001)$. (c) Probability plots for radiographic progression in relation to serum CRP levels in RA patients stratified by urinary ORM2 levels (high versus low ORM2).

Table 1 Multivariate logistic regression analysis for predicting radiographic progression using conventional risk factors plus urinary protein markers

\begin{tabular}{lll}
\hline Model & Odds ratio (95\% Cl) & P-value \\
\hline Model 1 (Basic model+ORM1, per 1-s.d. increase) & $1.27(0.90-1.79)$ & 0.166 \\
Model 2 (Basic model+ORM2, per 1-s.d. increase) & $1.25(1.01-1.54)$ & 0.044 \\
Model 3 (Basic model+GSN, per 1-s.d. increase) & $0.79(0.59-1.05)$ & 0.101 \\
Model 4 (Basic model+CD14, per 1-s.d. increase) & $1.00(0.99-1.00)$ & 0.501 \\
\hline
\end{tabular}

Abbreviations: $\mathrm{Cl}$, confidence interval; GSN, gelsolin; ORM, orosomucoid ( $\alpha 1$-acid glycoprotein).

Basic model included age, disease duration, C-reactive protein, anti-cyclic citrullinated peptide antibody positivity and glomerular filtration rate at baseline.

Logistic regression was used to obtain basic model-adjusted odds ratios for the presence of radiographic progression.

radiographic progression of $\mathrm{RA}(\mathrm{OR}=1.25,95 \% \mathrm{CI}$ : (1.01-1.54), $P=0.044$; Table 1), indicating that urinary ORM2 is an independent risk factor for RA progression. However, ORM1, sCD14 or GSN levels did not show a statistically significant association with radiographic progression (Table 1). To compare the diagnostic performance of urinary ORM2 for radiographic progression with that of serum CRP, we performed an ROC curve analysis using blood ESR/CRP and urine ORM2. Our results revealed that the
AUC for ORM2 was 0.792 (0.690-0.901; Figure 3b), which was comparable to the AUC for ESR (0.813 (0.734-0.889)), a conventional blood marker used to predict RA progression. ${ }^{19}$ The AUC for serum CRP was 0.890 (0.83-0.94).

\section{Complementary value of urinary ORM2 to current measures} for RA progression

We further investigated whether urinary ORM2 plus serum CRP can better predict radiographic progression than each 
individual parameter. To do this, we stratified patients based on urinary ORM2 concentrations. As shown in Figure 3c, patients with high urinary ORM2 $\left(\geqslant 2.21 \mathrm{ng} \mathrm{ml}^{-1}\right)$ showed a higher probability of radiographic progression than those with low urinary ORM2. The predicted probability of joint destruction at the median CRP level was 0.70 in the high ORM2 group and 0.22 in the low ORM2 group (Figure 3c). Based on these observations, we further analyzed whether urinary ORM2 could provide additional predictive value for radiographic progression when combined with serum CRP or ESR. To do this, we categorized RA patients into two groups based on positive levels for ORM, CRP and ESR (Table 2). The positive cutoff values were as follows: urinary ORM2 $>2.21 \mathrm{ng} \mathrm{ml}^{-1}$, CRP was $>0.3 \mathrm{mg} \mathrm{dl}^{-1}$, ESR for men $>$ age $/ 2 \mathrm{~mm} \mathrm{~h}^{-1}$ and ESR for women was $>($ age +10$) / 2 \mathrm{~mm} \mathrm{~h}^{-1}$. The results showed that RA patients who were positive for both ORM2 and serum CRP had significantly higher adjusted odds ratios (ORs) of radiographic progression than patients positive for a single marker or negative for both (Table 2). Patients who were positive for both ORM2 and ESR showed a similar trend. Specifically, in RA patients who were positive for both ORM2 and CRP $(n=43)$, the adjusted OR was significantly higher $(46.45$ (5.23-412.73), $P<0.001)$ compared with that of patients who were negative for both $(n=128)$. This $\mathrm{OR}$ was also much greater than the OR obtained by a conventional combinatory model with serum CRP and ESR $(\mathrm{OR}=4.09, P=0.039$; Table 2). A similar trend was found by a tree analysis to find the best combination of cutoff values to predict radiographic progression (Supplementary Figure 2). Taken together, these data suggest that urinary ORM2 can offer complementary dimensions to current serum parameters for RA activity and

Table 2 Multivariate logistic regression analysis of a combination of urinary proteins with blood markers for predicting radiographic progression

\begin{tabular}{|c|c|c|}
\hline & Odds ratio $(95 \% \mathrm{Cl})$ & P-value \\
\hline \multicolumn{3}{|c|}{ Model+ORM2-CRP combination } \\
\hline Negative $(n=128)$ & 1 & \\
\hline One positive $(n=93)$ & $8.72(1.01-77.68)$ & 0.048 \\
\hline Two positive $(n=43)$ & $46.45(5.23-412.73)$ & 0.001 \\
\hline \multicolumn{3}{|c|}{ Model+ORM2-ESR combination } \\
\hline Negative $(n=85)$ & 1 & \\
\hline One positive $(n=110)$ & $1.09(0.25-4.84)$ & 0.309 \\
\hline Two positive $(n=69)$ & $3.94(1.10-15.69)$ & 0.042 \\
\hline \multicolumn{3}{|c|}{ Model+CRP-ESR combination } \\
\hline Negative $(n=90)$ & 1 & \\
\hline One positive $(n=84)$ & $1.70(0.35-3.84)$ & 0.268 \\
\hline Two positive $(n=90)$ & $4.09(1.07-15.55)$ & 0.039 \\
\hline
\end{tabular}

prognosis. It has an even better predictive power for RA progression than a combined measure of ESR and CRP.

\section{DISCUSSION}

The goal of RA treatment is to maintain minimal residual disease levels or to achieve complete remission. ${ }^{1,20}$ Because the DAS28 score is one of the best measures to determine RA activity, rheumatologists measure DAS28 scores routinely and use it as one of the justifications for recommending a change in treatment. ${ }^{20}$ However, RA activity fluctuates over time, and DAS28 scores in patients who transiently improved just before the patient's visit may be misleadingly low and the actual mean activity between the visits high. Therefore, regular selfmonitoring of RA activity and the identification of surrogate markers is required so that actual RA activity correlates with DAS28. Urine can be collected daily at home, non-invasively in large quantities, and urine is more stable than other biofluids. ${ }^{21}$ It may serve as a potentially rich source of biomarkers that can indicate systemic inflammation through the excretion of overproduced proteins. ${ }^{22}$ Here, we introduced an innovative approach using the patients' urine to assess the activity and progression of RA. We found that three urinary biomarker candidates-ORM1, ORM2 and sCD14-correlated well with RA activity. Importantly, urinary ORM2 levels showed the highest correlation with DAS28 scores, which was comparable to that of serum CRP. Given that urinary ORM2 had an AUC comparable to that of serum CRP for patients with high RA activity (DAS28 $>5.1$ ), it might be able to replace blood measures for RA activity, thus eliminating the requirement for blood sampling.

RA is a multifaceted disease in which the evaluation of any one of the available measures does not always provide reliable information on a patient's status. ${ }^{23-25}$ The fact that there are more than 63 currently available measurement tools for RA disease activity reflects the efforts to search for models that could best describe this disease's status. ${ }^{26}$ In addition, because of the pathological complexity of $\mathrm{RA}^{23}$ a single aspect of disease activity may not be sufficient in most measurement indices. Rather, combinations of several biomarkers might offer the possibility to enhance diagnostic accuracy. Here, we found that the combinatory analysis of two biomarkers out of the set of ORM1, ORM2 and sCD14 in the urine of RA patients showed a better diagnostic sensitivity and specificity in predicting high RA activity (DAS28 $>5.1$ ) than using a single biomarker. Specifically, for RA patients with high levels of ORM1 and ORM2 in their urine, the sensitivity and specificity for predicting high RA activity were $98 \%$ and $76 \%$, respectively. Elevated CRP alone $\left(>0.3 \mathrm{mg} \mathrm{dl}^{-1}\right)$ showed rates of $75 \%$ and $60 \%$ for the sensitivity and specificity, respectively, of predicting high RA activity (data not shown). These results suggest that urine tests using the above biomarker sets could better discriminate active RA from inactive RA without the need for blood tests, such as those for ESR and CRP. The present study provides a rationale to develop urinary kits that can be utilized at home for self-assessment of RA activity. Similarly, urinary ORM1, ORM2 and sCD14 
might offer new diagnostic targets for other forms of chronic arthritis.

The accumulated disease activity of RA mainly affects RA prognosis and extra-articular complications, such as atherosclerosis. $1,27,28$ Our results demonstrating the association of ORM1, ORM2 and sCD14 with RA activity prompted us to investigate the role of these biomarkers in predicting RA progression. Multivariate logistic regression analysis was performed to determine whether ORM1, ORM2 and SCD14 could predict radiographic progression after 3 years. Our results showed that urinary ORM2, but not ORM1 or sCD14, was an independent risk factor for the prediction of the radiographic progression of RA (Table 1). ORM2 could provide additional benefits to the use of current blood markers ESR and CRP (Table 2). Importantly, in patients who had elevated levels of both ORM2 ( $\geqslant 2.21 \mathrm{ng} \mathrm{ml}^{-1}$ ) and CRP $\left(>0.3 \mathrm{mg} \mathrm{dl}^{-1}\right)$, the adjusted OR was 46.5. These data clearly show that urinary ORM2, as a non-invasive diagnostic biomarker, could be a promising parameter for the prediction of the radiographic progression of RA. It also may offer complementary dimensions to the current serum parameters used for RA prognosis.

In summary, in a single-center prospective study, we found that urinary ORM1, ORM2 and sCD14 levels were elevated in RA patients and correlated well with the status of the disease activity. In particular, the use of urine tests for ORM1, ORM2 and sCD14 (without the need for blood ESR/CRP tests) efficiently represented the presence of high RA activity. In parallel, a more rapid radiographic progression over 3 years was observed in patients with higher ORM2 levels. Furthermore, the combination of urinary ORM2 and serum CRP synergistically increased the predictability of radiographic progression. Our data provide evidence that blood-free urinary biomarkers are promising surrogates for assessing disease activity and prognosis of RA. We anticipate that our urinary biomarkers will provide novel candidates for patient-driven measurements of RA activity at home and may shift the paradigm from blood to urine testing in the assessment of RA activity and prognosis in hospitals. We are currently developing a new diagnostic kit and portable machine (FREND System at http://www.nanoentek.com) to accurately measure concentrations of ORM1, ORM2 and $\mathrm{SCD} 14$ in a drop of urine within 10 min (Supplementary Figure 3).

\section{CONFLICT OF INTEREST}

The authors declare no conflict of interest.

\section{ACKNOWLEDGEMENTS}

We thank all members of the Institute of Bone and Joint Diseases at the Catholic University of Korea. This research was supported by Basic Science Research Program through the National Research Foundation of Korea (NRF) funded by the Ministry of Education (2014R1A6A3A04054066 and NRF-2014R1A1A2055850). This work was supported by grants from the Korea Healthcare Technology R\&D Project, Ministry for Health, Welfare and Family Affairs (No. HI14C3417), the National Research Foundation of Korea (NRF) funded by the Ministry of Education, Science and Technology (2014R1A2A1A11049812 and 2015R1A3A2032927) and the Catholic Medical Center Research Foundation made in the 2014 program year.

1 Smolen JS, Aletaha D, Bijlsma JW, Breedveld FC, Boumpas D, Burmester G et al. Treating rheumatoid arthritis to target: recommendations of an international task force. Ann Rheum Dis 2010; 69: 631-637.

2 Wells G, Li T, Maxwell L, Maclean R, Tugwell P. Responsiveness of patient reported outcomes including fatigue, sleep quality, activity limitation, and quality of life following treatment with abatacept for rheumatoid arthritis. Ann Rheum Dis 2008; 67: 260-265.

3 Böttiger LE, Svedberg CA. Normal erythrocyte sedimentation rate and age. Br Med J 1967; 2: 85-87.

4 Kushner I. C-reactive protein in rheumatology. Arthritis Rheum 1991; 34: 1065-1068.

5 Pincus T, Braun J, Kavanaugh A, Smolen JS. Optimisation of assessment for rheumatic diseases in clinical trials, observational studies and routine clinical care. Clin Exp Rheumatol 2014; 32: S-1.

6 Nell VP, Machold KP, Stamm TA, Eberl G, Heinzl H, Uffmann M et al. Autoantibody profiling as early diagnostic and prognostic tool for rheumatoid arthritis. Ann Rheum Dis 2005; 64: 1731-1736.

7 Watson L, Beresford MW. Urine biomarkers in juvenile-onset SLE nephritis. Pediatr Nephrol 2013; 28: 363-374.

8 Matheson A, Willcox MD, Flanagan J, Walsh BJ. Urinary biomarkers involved in type 2 diabetes: a review. Diabetes Metab Res Rev 2010; 26: 150-171.

9 Kang MJ, Park YJ, You S, Yoo SA, Choi S, Kim DH et al. Urinary proteome profile predictive of disease activity in rheumatoid arthritis. J Proteome Res 2014; 13: 5206-5217.

10 Arnett FC, Edworthy SM, Bloch DA, McShane DJ, Fries JF, Cooper NS et al. The American Rheumatism Association 1987 revised criteria for the classification of rheumatoid arthritis. Arthritis Rheum 1988; 31: 315-324.

11 Prevoo ML, van 't Hof MA, Kuper HH, van Leeuwen MA, van de Putte LB, van Riel PL. Modified disease activity scores that include twenty-eightjoint counts. Development and validation in a prospective longitudinal study of patients with rheumatoid arthritis. Arthritis Rheum 1995; 38: 44-48.

12 van der Heijde D. How to read radiographs according to the Sharp/van der Heijde method. J Rheumatol 2000; 27: 261-263.

13 Giles JT, van der Heijde DM, Bathon JM. Association of circulating adiponectin levels with progression of radiographic joint destruction in rheumatoid arthritis. Ann Rheum Dis 2011; 70: 1562-1568.

14 Horneff G, Sack U, Kalden JR, Emmrich F, Burmester GR. Reduction of monocyte-macrophage activation markers upon anti-CD4 treatment. Decreased levels of IL-1, IL-6, neopterin and soluble CD14 in patients with rheumatoid arthritis. Clin Exp Immunol 1993; 91: 207-213.

15 Yu S, Nakashima N, Xu BH, Matsuda T, Izumihara A, Sunahara N et al. Pathological significance of elevated soluble CD14 production in rheumatoid arthritis: in the presence of soluble CD14, lipopolysaccharides at low concentrations activate RA synovial fibroblasts. Rheumatol Int 1998; 17: 237-243.

16 Mottonen T, Paimela L, Leirisalo-Repo M, Kautiainen H, Ilonen J, Hannonen P. Only high disease activity and positive rheumatoid factor indicate poor prognosis in patients with early rheumatoid arthritis treated with "sawtooth" strategy. Ann Rheum Dis 1998; 57: 533-539.

17 Wolfe $F$. The prognosis of rheumatoid arthritis: assessment of disease activity and disease severity in the clinic. Am J Med 1997; 103 : $12 \mathrm{~s}-18 \mathrm{~s}$.

18 Lindqvist E, Eberhardt K, Bendtzen K, Heinegard D, Saxne T. Prognostic laboratory markers of joint damage in rheumatoid arthritis. Ann Rheum Dis 2005; 64: 196-201.

19 Plant MJ, Williams AL, O'Sullivan MM, Lewis PA, Coles EC, Jessop JD. Relationship between time-integrated C-reactive protein levels and radiologic progression in patients with rheumatoid arthritis. Arthritis Rheum 2000; 43: 1473-1477.

20 Smolen J, Landewé R, Breedveld FC, Buch M, Burmester G, Dougados M et al. EULAR recommendations for the management of rheumatoid arthritis with synthetic and biological disease-modifying antirheumatic drugs: 2013 update. Ann Rheum Dis 2014; 73: 492-509. 
21 Decramer S, Gonzalez de Peredo A, Breuil B, Mischak H, Monsarrat B, Bascands JL et al. Urine in clinical proteomics. Molecular \& cellular proteomics. Mol Cell Proteomics 2008; 7: 1850-1862.

22 Barratt J, Topham P. Urine proteomics: the present and future of measuring urinary protein components in disease. CMAJ 2007; 177: 361-368.

23 Firestein GS. Evolving concepts of rheumatoid arthritis. Nature 2003; 423: 356-361.

24 Aletaha D, Nell VP, Stamm T, Uffmann M, Pflugbeil S, Machold K et al. Acute phase reactants add little to composite disease activity indices for rheumatoid arthritis: validation of a clinical activity score. Arthritis Res Ther 2005; 7: R796-R806.

25 Smolen JS, Breedveld FC, Schiff MH, Kalden JR, Emery P, Eberl G et al. A simplified disease activity index for rheumatoid arthritis for use in clinical practice. Rheumatology (Oxford) 2003; 42: 244-257.

26 Anderson J, Caplan L, Yazdany J, Robbins ML, Neogi T, Michaud K et al. Rheumatoid arthritis disease activity measures: American College of Rheumatology recommendations for use in clinical practice. Arthritis Care Res (Hoboken) 2012; 64: 640-647.
27 Puttevils D, De Vusser P, Geusens P, Dens J. Increased cardiovascular risk in patients with rheumatoid arthritis: an overview. Acta Cardiol 2014; 69: $111-118$.

28 Goodman SM, Mackenzie CR. Cardiovascular risk in the rheumatic disease patient undergoing orthopedic surgery. Curr Rheumatol Rep 2013; 15: 354.

(c) (1) (2)(2) This work is licensed under a Creative Commons Attribution-NonCommercial-ShareAlike 4.0 International License. The images or other third party material in this article are included in the article's Creative Commons license, unless indicated otherwise in the credit line; if the material is not included under the Creative Commons license, users will need to obtain permission from the license holder to reproduce the material. To view a copy of this license, visit http:// creativecommons.org/licenses/by-nc-sa/4.0/

Supplementary Information accompanies the paper on Experimental \& Molecular Medicine website (http://www.nature.com/emm) 